\title{
Correction to: Editorial re: Facial nerve function and hearing after microsurgical removal of sporadic vestibular schwannomas in a population-based cohort by Ismail Taha et al.
}

\section{Martin Sames ${ }^{1}$}

Published online: 4 December 2019

(C) Springer-Verlag GmbH Austria, part of Springer Nature 2019

\section{Correction to: Acta Neurochirurgica}

$$
\text { https://doi.org/10.1007/s00701-019-04092-Z }
$$

The correct name of the author is Martin Sames and not Michael.

The correct authorname should be:

$<$ GivenName $>$ Martin $</$ GivenName $>$

$<$ FamilyName $>$ Sames $<$ /FamilyName $>$

The Publisher regrets this error.

The original article has been corrected.

The online version of the original article can be found at https://doi.org/ 10.1007/s00701-019-04092-z

Martin Sames

martin.sames@kzcr.eu

1 Masaryk Hospital, University J.E.Purkyne, Usti nad

Labem, Czech Republic 